\title{
A Time for MYC: Metabolism and Therapy
}

\author{
Chi V. DANG \\ Abramson Cancer Center, Perelman School of Medicine, University of Pennsylvania, \\ Philadelphia, Pennsylvania 19104 \\ Correspondence: dangvchi@upenn.edu
}

\begin{abstract}
The MYC oncogene is frequently deregulated in human cancers, whereas the proto-oncogene is exquisitely, tightly regulated in normal cells. Deregulated MYC drives transcriptional imbalance, thereby altering metabolism and disrupting the circadian Bmal1-Clock E-box-dependent transcriptional circuitry. Sustained oncogenic MYC expression drives a constitutive growth program with mammalian target of rapamycin (mTOR) activation that renders cells dependent on nutrients, such that glucose or glutamine deprivation could trigger cell death and key enzymes such as lactate dehydrogenase A (LDHA) and glutaminase (GLS) amenable for targeting in cancers. Further, MYC-mediated suppression of the circadian clock is surmised to suspend the inhibitory effect of Bmal1-Clock on metabolism, allowing for MYC-driven cancer cells to reach a higher state of anabolic metabolism. Hence, metabolic therapy could be deployed, particularly at specific times of the day, to diminish side effects to normal tissues while maintaining antitumor efficacy.
\end{abstract}

Signals, which stimulate cell growth leading to cell division, emanate from growth factor receptor signaling and nutrient sensors that together amplify growth signals. Growth factor and nutrient sensing signaling stimulate mammalian target of rapamycin (mTOR) and MYC expression (Laplante and Sabatini 2012; Stine et al. 2015), which together transmit growth signals by amplifying translation and transcription to generate transcripts that are in turn translated to build components of the growing cell (Fig. 1). MYC stimulates transcription by binding to open chromatin and largely through releasing paused RNA polymerases (Kress et al. 2015; Wolf et al. 2015). As such, "housekeeping" metabolic genes and genes involved in mitochondrial function and ribosome biogenesis are accessible by MYC, which readily activates these genes through E-boxes in open chromatin found in virtually all nucleated cells that universally use core metabolic pathways such as glycolysis and glutaminolysis (Fig. 2; Dang 2012, 2013). Distinct from physiologic levels of MYC, a high oncogenic level of MYC is surmised to bind loweraffinity DNA binding sites, such as those bound by other Ebox binding transcription factors (Clock-Bmal1, Srebp, or hypoxia-inducible factors [HIFs]), and bind enhancer sequences, resulting in nonlinear amplification of transcripts (Fig. 2; Dang 2012). The imbalanced transcriptional program is likely to result in nonstoichiometric production of RNAs or proteins in multicomponent complexes and protein overload and hence is responsible for integrated stress responses in oncogenic MYC-driven cells (Hart et al. 2012; Cunningham et al. 2014). This stress condition also provides a therapeutic window.

Hence, the MYC transcriptional response program is characterized by metabolic reprogramming that is shown experimentally through isotopic tracer studies using la- beled glucose or glutamine. In the model of human Burkitt lymphoma with an inducible MYC gene, both glycolysis and glutaminolysis were stimulated by MYC that also sustains glutamine metabolism under hypoxic conditions (Fig. 3; Le et al. 2012; Murphy et al. 2013). These observations suggest that pathways involved in glucose and glutamine metabolism could be targeted for cancer therapy (Fig. 4). Indeed, proof-of-concept studies provided evidence that inhibition of lactate dehydrogenase, the key enzyme involved in the Warburg effect, and glutaminase could delay tumorigenesis (Le et al. 2010; Xie et al. 2014; Xiang et al. 2015). However, use of a single metabolic therapeutic agent can result in metabolic rewiring and subsequent progression of resistant disease (Yuneva et al. 2012; Davidson et al. 2016). As such, deeper understanding of metabolic rewiring driven by specific oncogenic drivers (MYC, MET, p53, PTEN, PI3 K, Ras, and Akt) in specific tissues is essential for the future use of metabolic therapy, which is likely most effective in strategic combination therapies.

We surmise that high levels of oncogenic MYC could perturb the circadian clock by MYC's invasion of clock driven E-boxes (Fig. 5; Altman et al. 2015). The molecular clock is driven by the central clock Clock-Bmal1 transcriptional heterodimer, which regulates clock-controlled genes as well as induces two negative feedback loops that provide the oscillatory activity of Clock-Bmall. One feedback loop involves posttranscriptional regulation by the Clock-Bmal1 target genes CRYs and PERs. The other involves the induction of REV-ERBs, which transcriptionally repress the expression of Bmal1. Using an inducible MYC system in a U2OS cell line that bears the Bmall promoter-luciferase reporter, we document the ability of MYC to disrupt and suppress Bmal1 expression (Fig. 5). (C) 2016 Dang. This article is distributed under the terms of the Creative Commons Attribution-NonCommercial License, which permits reuse and
redistribution, except for commercial purposes, provided that the original author and source are credited. 


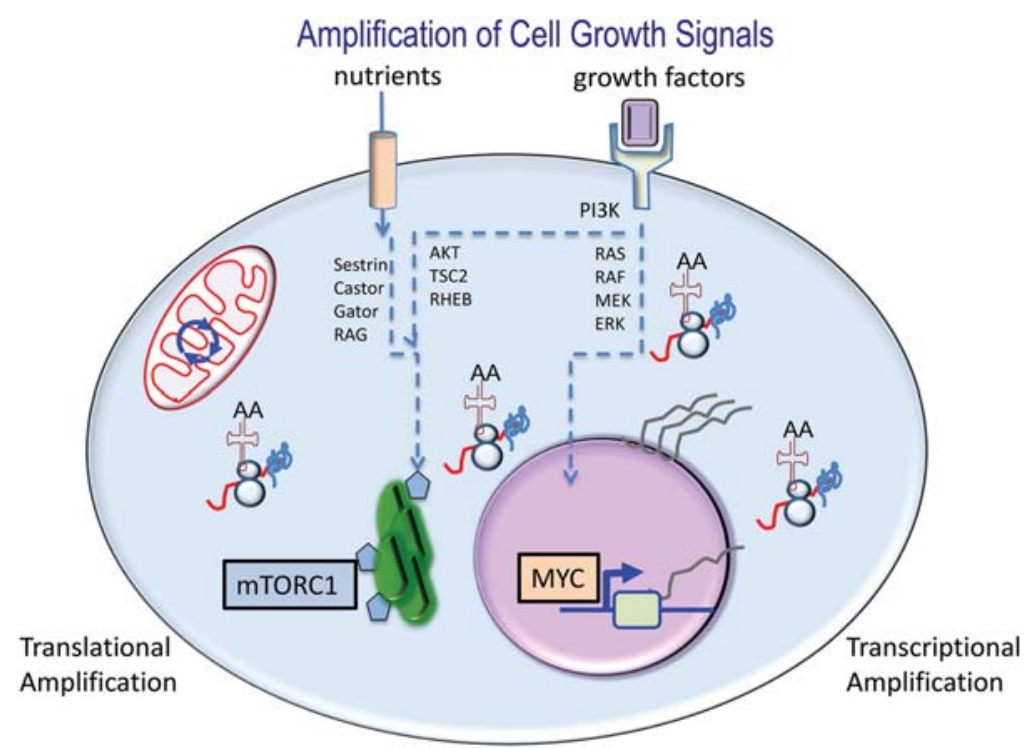

Figure 1. Amplification of cell growth signals. The diagram depicts the dependency of growing mammalian cells on nutrients, which are imported by transporters, and growth factors, which are sensed by growth factor receptors. Nutrients, particularly amino acids, are sensed along with growth factor signaling through tuberous sclerosis complex 2 (TSC2) and AKT resulting in the activation of mammalian target of rapamycin complex 1 (mTORC1), which increases the cell's protein synthetic capacity resulting in translational amplification of growth and nutrient signaling. On the other hand, growth factor signaling through phosphoinositide 3-kinase (PI3K), RAS-RAF-MEK-ERK pathways together with nutrient sensing triggers transcriptional amplification of growth through activation of MYC, a key transcriptional factor for cell growth.

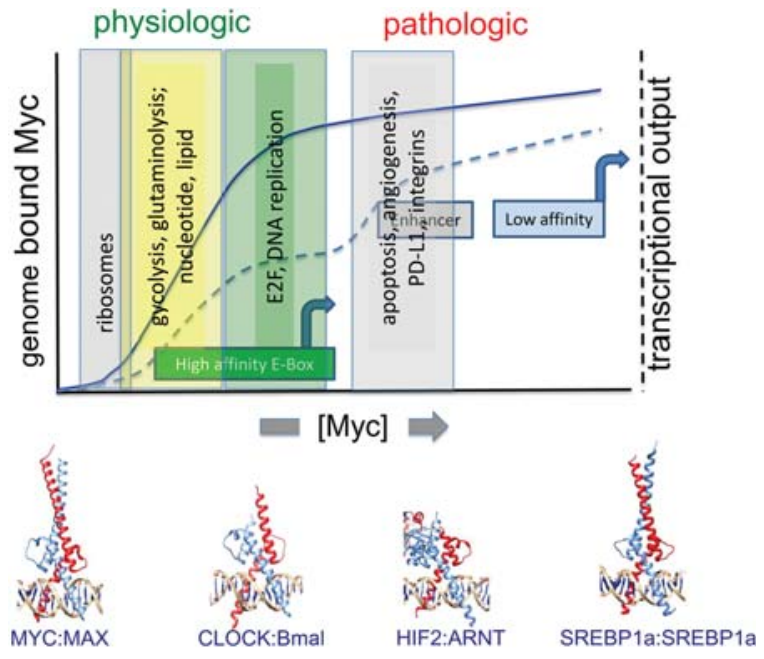

Figure 2. A graph illustrating that increased MYC levels result in the binding of high-affinity E-box sites and activation of "housekeeping genes" such as those involved in ribosome biogenesis or central metabolic pathways (glycolysis, glutaminolysis). With cell growth reaching a critical size, MYC collaborates with E2F transcription factors for cell entry in S phase for DNA replication. When MYC is deregulated, it could ectopically bind enhancer sequences and low-affinity sites, which result in imbalanced transcriptional amplification triggering apoptosis, angiogenesis, or activation of immune checkpoints through specific "ectopic" target gene expression. The low-affinity sites may consist of E-boxes that are targets of other E-box binding transcription factors shown at the bottom of the figure.
These studies show that E-box-driven negative regulators (Crys, Pers, and Rev-erbs) were all activated by ectopic MYC, whereas Bmall expression was diminished in a fashion that could be partially rescued by knocking down Rev-erb expression. Subsequent to our findings, a report corroborated that MYC could suppress Bmall expression and the clock, but it implicates a role for direct suppression of Clock and Bmall expression via Mizl (Shostak et al. 2016). Hence, MYC consistently suspends the clock, but this is likely to involve a direct Miz1-dependent suppression and an indirect suppression of Bmall via MYC induction of Rev-erbs.

Based on the observation that MYC could suspend the clock and drive anabolic metabolism, we hypothesize that effective anticancer metabolic therapy may depend on the time of drug administration during the day - particularly for short-lived drugs (Fig. 6). We hypothesize that toxicity to normal tissues, which have oscillatory metabolic gene expression and circadian metabolism, would be reduced depending on the time of day of drug administration, whereas efficacy against MYC-driven tumors would be retained because the tumors have constitutive high expression of the target gene in question such as NAMPT, which is a target gene of both Clock-Bmal1 in normal tissues and oncogenic MYC in MYC-driven cancers. As such, we examine the levels on $\mathrm{NAD}^{+}$and expression of NAMPT, a rate-limiting enzyme for $\mathrm{NAD}^{+}$synthesis, and found that ectopic MYC induced a sustained expression of 


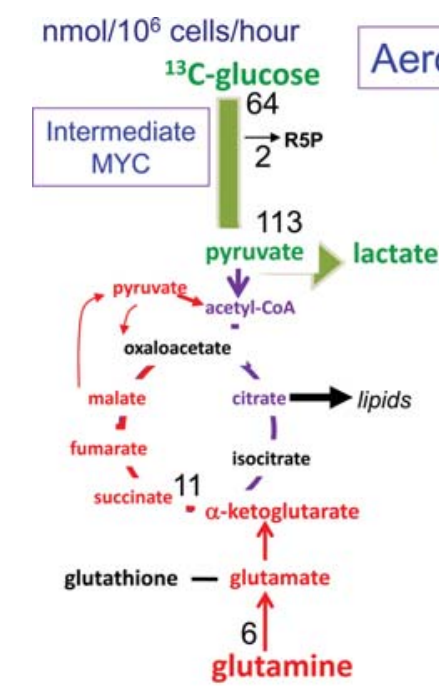

Figure 3. The cartoon summarizes the flux of metabolites through glycolysis and the TCA cycle as determined by isotopic labeling of a MYC-inducible human B-cell lymphoma model. The two metabolic diagrams on the left illustrate the findings of Murphy et al. (2013) comparing intermediate MYC expression under a nontumorigenic state with ectopic high MYC expression under a tumorigenic state. Note that the thickness of the arrows approximates flux, which is shown as numbers with values of nmol $/ 10^{6} \mathrm{cells} / \mathrm{h}$. Although ectopic high MYC increased glycolytic flux by $20 \%$, the flux through the TCA cycle increased by $400 \%$. The metabolic diagram on the right summarizes the findings of Le et al. (2012) indicating that hypoxic high MYC expressing B lymphocytes had increased production of lactate from glucose and continued glutamine flux into the TCA cycle as well as increased glutathione production under hypoxia.

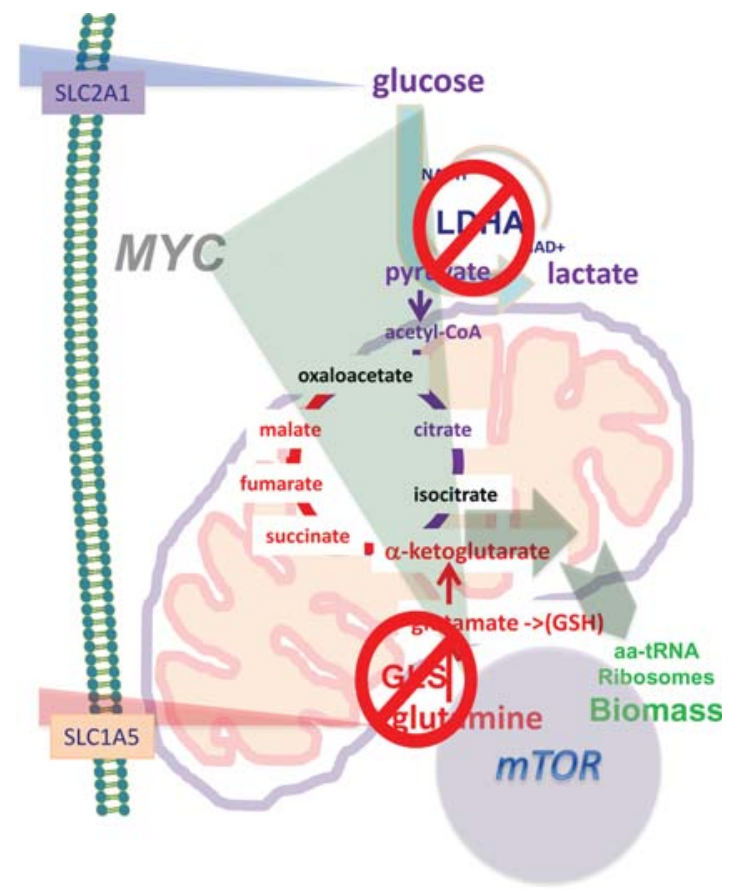

Figure 4. The diagram depicts the ability of ectopic MYC expression to drive the expression of genes involved in glucose and glutamine transport, glycolysis, glutaminolysis, and protein synthesis (aa-tRNA, aminoacyl-tRNAs). Together with mammalian target of rapamycin (mTOR), MYC drives deregulated cell growth rendering cells dependent on key metabolic enzymes such as lactate dehydrogenase A (LDHA) and glutaminase (GLS), which are marked as being targeted for proof of concept that metabolic inhibitors could curb tumor growth.
NAMPT accompanied by high levels of intracellular $\mathrm{NAD}^{+}$. To assess the potential importance of $\mathrm{NAD}^{+}$dysregulation by MYC, we studied a potent inhibitor of NAMPT, FK866, that is effective against in a xenograft model of human Burkitt lymphoma (Le et al. 2010). Because NAMPT is expressed with remarkable circadian oscillation with nadirs in the morning and peaks in early evening in mouse livers, we empirically chose to treat animals at 10 a.m. or 6 p.m. FK 866 has been under clinical development and clinical trials uncovered a dose-limiting toxicity of low platelet counts or thrombocytopenia in patients (Sampath et al. 2015). As such, we hypothesized that FK866 given at two different times of the day would result in similar efficacy but distinctly difference toxicities. Indeed, we found that administration of FK866 at 6 p.m., but not 10 a.m., was associated with thrombocytopenia, but antitumor efficacy was indistinguishable. These observations suggest that suspension of the clock by MYC could provide a new therapeutic window for timed metabolic therapies and those metabolic therapies could be affected by the circadian biology of normal tissues and cancer cells.

\section{ACKNOWLEDGMENTS}

The work described herein was all performed by many members of the Dang lab and supported by the National Cancer Institute, Leukemia Lymphoma Society, and Stand-Up-to-Cancer. This short overview is a summary of the presentation given at the 81st CSHL Quantitative Biology Meeting and is not meant to be an exhaustive 

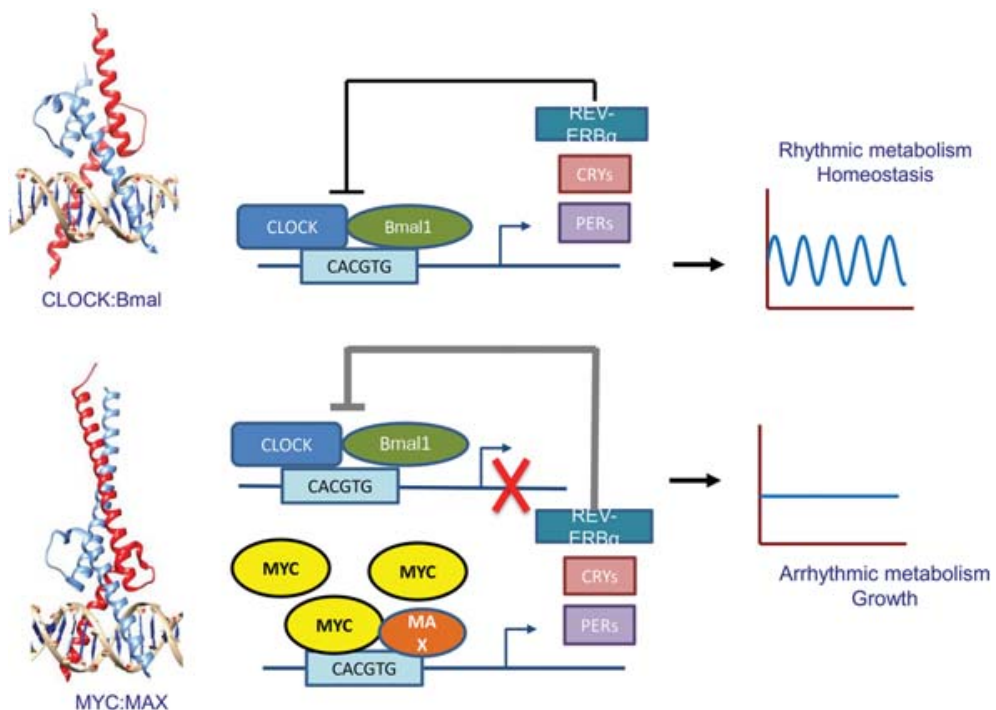

Figure 5. The cartoons illustrate the circadian transcriptional clock machinery (top) and its putative disruption by ectopic MYC expression (bottom). The central transcription factor Clock-Bmall (top) induces two negative feedback loops via the induction of CRYs and PERs (posttranscriptional negative feedback) and REV-REVs (transcriptional negative feedback), which create the oscillatory activity of Clock-Bmall that drives circadian expression of target metabolic genes. Ectopic MYC (bottom) is depicted to bind Clock-driven E-boxes to activate the negative regulators constitutively that resulted in continuous suppression of Bmal1 expression and inhibit circadian expression of Clock target genes. Crystal structures of Clock-Bmall and Myc-Max are shown on the left, illustrating the structural similarities of these helix-loop-helix transcription factors and their binding to E-boxes (5'-CACGTG-3').

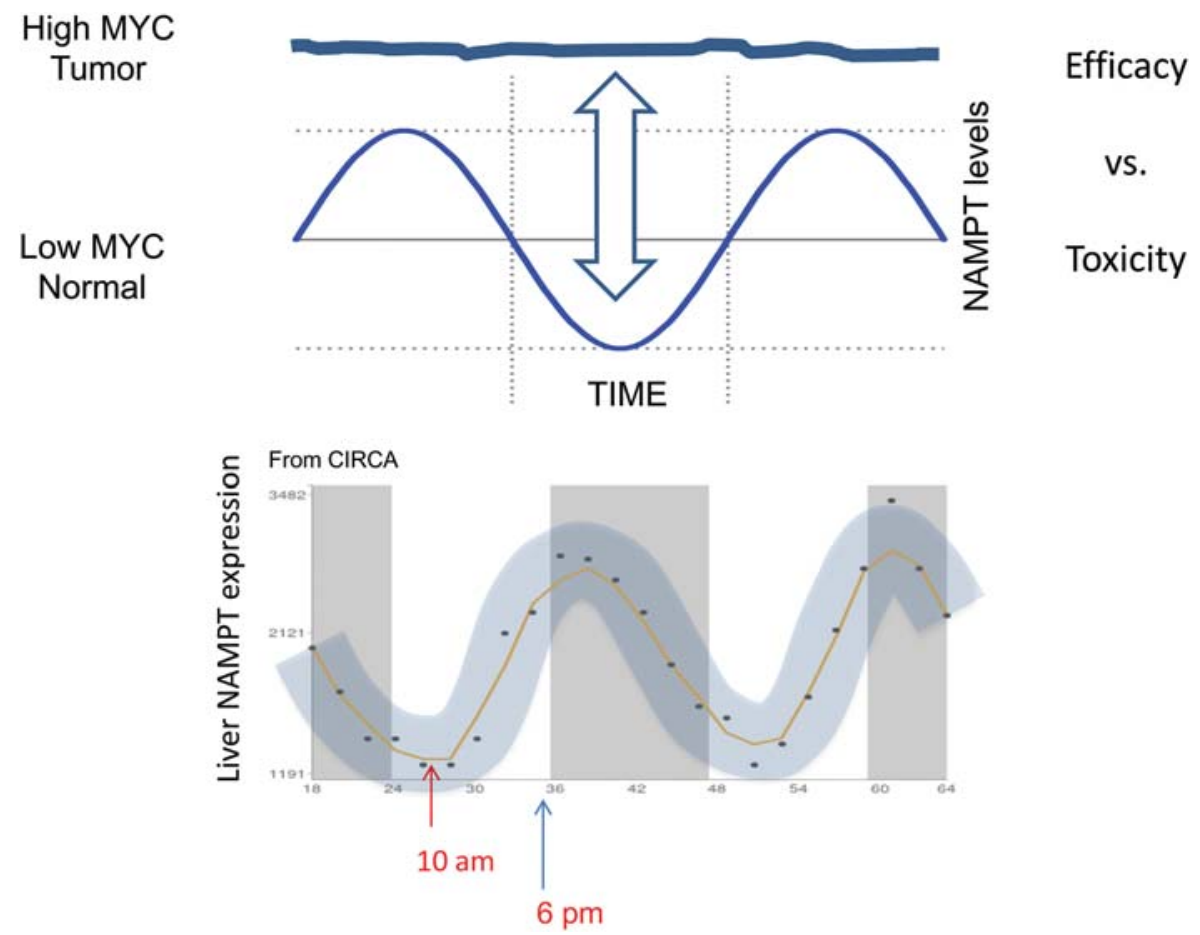

Figure 6. The figure illustrates the hypothesis that MYC deregulation of metabolic genes that are regulated normally by the ClockBmal1 transcription factor, such as nicotinamide phosphoribosyltransferase (NAMPT), could open up a therapy window for metabolic cancer therapy. It is surmised that toxicity to normal tissues, which have oscillatory metabolic gene expression and circadian metabolism, would be reduced depending on the time of day of drug administration, whereas efficacy against MYC-driven tumors would be retained because the tumors have constitutive high expression of the target gene in question such as NAMPT, which is a target gene of both Clock-Bmal1 (in normal tissues, such as liver shown at the bottom) and oncogenic MYC. 
review of the literature, which is enriched by many papers not cited here.

\section{REFERENCES}

Altman BJ, Hsieh AL, Sengupta A, Krishnanaiah SY, Stine ZE, Walton ZE, Gouw AM, Venkataraman A, Li B, GorakshaHicks P, et al. 2015. MYC disrupts the circadian clock and metabolism in cancer cells. Cell Metab 22: 1009-1019.

Cunningham JT, Moreno MV, Lodi A, Ronen SM, Ruggero D. 2014. Protein and nucleotide biosynthesis are coupled by a single rate-limiting enzyme, PRPS2, to drive cancer. Cell 157: $1088-1103$.

Dang CV. 2012. MYC on the path to cancer. Cell 149: 22-35.

Dang CV. 2013. MYC, metabolism, cell growth, and tumorigenesis. Cold Spring Harb Perspect Med 3: a014217.

Davidson SM, Papagiannakopoulos T, Olenchock BA, Heyman JE, Keibler MA, Luengo A, Bauer MR, Jha AK, O'Brien JP, Pierce KA, et al. 2016. Environment impacts the metabolic dependencies of Ras-driven non-small cell lung cancer. Cell Metab 23: 517-528.

Hart LS, Cunningham JT, Datta T, Dey S, Tameire F, Lehman SL, Qiu B, Zhang H, Cerniglia G, Bi M, et al. 2012. ER stressmediated autophagy promotes Myc-dependent transformation and tumor growth. J Clin Invest 122: 4621-4634.

Kress TR, Sabò A, Amati B. 2015. MYC: Connecting selective transcriptional control to global RNA production. Nat Rev Cancer 15: 593-607.

Laplante M, Sabatini DM. 2012. mTOR signaling in growth control and disease. Cell 149: 274-293.

Le A, Cooper CR, Gouw AM, Dinavahi R, Maitra A, Deck LM, Royer RE, Vander Jagt DL, Semenza GL, Dang CV. 2010.
Inhibition of lactate dehydrogenase A induces oxidative stress and inhibits tumor progression. Proc Natl Acad Sci 107: 2037-2042.

Le A, Lane AN, Hamaker M, Bose S, Gouw A, Barbi J, Tsukamoto T, Rojas CJ, Slusher BS, Zhang H, et al. 2012. Glucoseindependent glutamine metabolism via TCA cycling for proliferation and survival in B cells. Cell Metab 15: 110-121.

Murphy TA, Dang CV, Young JD. 2013. Isotopically nonstationary ${ }^{13} \mathrm{C}$ flux analysis of Myc-induced metabolic reprogramming in B-cells. Metab Eng 15: 206-217.

Sampath D, Zabka TS, Misner DL, O'Brien T, Dragovich PS. 2015. Inhibition of nicotinamide phosphoribosyltransferase (NAMPT) as a therapeutic strategy in cancer. Pharmacol Ther 151: $16-31$.

Shostak A, Ruppert B, Ha N, Bruns P, Toprak UH; ICGC MMML-Seq Project, Eils R, Schlesner M, Diernfellner A, Brunner M. 2016. MYC/MIZ1-dependent gene repression inversely coordinates the circadian clock with cell cycle and proliferation. Nat Commun 7: 11807.

Stine ZE, Walton ZE, Altman BJ, Hsieh AL, Dang CV. 2015. MYC, metabolism, and cancer. Cancer Discov 5: 1024-1039.

Wolf E, Lin CY, Eilers M, Levens DL. 2015. Taming of the beast: Shaping Myc-dependent amplification. Trends Cell Biol 25: 241-248.

Xiang Y, Stine ZE, Xia J, Lu Y, O'Connor RS, Altman BJ, Hsieh AL, Gouw AM, Thomas AG, Gao P, et al. 2015. Targeted inhibition of tumor-specific glutaminase diminishes cell-autonomous tumorigenesis. J Clin Invest 125: 2293-2306.

Xie H, Hanai J, Ren JG, Kats L, Burgess K, Bhargava P, Signoretti S, Billiard J, Duffy KJ, Grant A, et al. 2014. Targeting lactate dehydrogenase A inhibits tumorigenesis and tumor progression in mouse models of lung cancer and impacts tumor-initiating cells. Cell Metab 19: 795-809. 


\section{$\$_{\text {CSH\& }}^{\infty}$ Cold Spring Harbor Symposia SYMPOSIA}

\section{A Time for MYC: Metabolism and Therapy}

Chi V. Dang

Cold Spring Harb Symp Quant Biol 2016 81: 79-83 originally published online February 7, 2017 Access the most recent version at doi:10.1101/sqb.2016.81.031153

References This article cites 17 articles, 3 of which can be accessed free at: http://symposium.cshlp.org/content/81/79.full.html\#ref-list-1
Creative This article is distributed under the terms of the
Commons http://creativecommons.org/licenses/by-nc/4.0/, which permits reuse and License redistribution, except for commercial purposes, provided that the original author and source are credited.

Email Alerting
Service $\quad \begin{aligned} & \text { Receive free email alerts when new articles cite this article - sign up in } \\ & \text { the box at the top right corner of the article or click here. }\end{aligned}$ 